\title{
Protection via a ROM4 DNA vaccine and peptide against Toxoplasma gondii in BALB/c mice
}

Yali Han ${ }^{1}$, Aihua Zhou', Gang Lu', Guanghui Zhao ${ }^{3}$, Lin Wang ${ }^{4}$, Jingjing Guo ${ }^{1}$, Pengxia Song ${ }^{1}$, Jian Zhou', Huaiyu Zhou', Hua Cong ${ }^{1}$ and Shenyi He 1* $^{*}$

\begin{abstract}
Background: Toxoplasma gondii (T. gondii) is an obligate intracellular protozoan parasite with a broad host range including most warm-blooded animals, including humans. T. gondii surface antigen 1 (SAG1) is a well-characterized T. gondii antigen. T. gondii expresses five nonmitochondrial rhomboid intramembrane proteases, TgROM1-5. TgROM4 is uniformly distributed on the surface of T. gondii and involved in regulating MIC2, MIC3, MIC6, and AMA1 during T. gondii invasion of host cells. Bioinformatics have predicted ROM4 B-cell and T-cell epitopes. Immunization strategy is also a key factor in determining the effectiveness of the immune response and has gained increasing attention in T. gondii vaccine research. In this study, we used a DNA prime-peptide boost vaccination regimen to assess the protective efficacy of various vaccination strategies using TgROM4.
\end{abstract}

Methods: We identified a polypeptide (YALLGALIPYCVEYWKSIPR) using a bioinformatics approach, and immunized mice using a DNA-prime and polypeptide-boost regimen. BALB/c mice were randomly divided into six groups, including three experimental groups (peptide, pROM4 and pROM4/peptide) and three control groups (PBS, pEGFP$\mathrm{C} 1$ and pSAG1). Mice were then immunized intramuscularly four times. After immunization, lgG and cytokine productions were determined using enzyme-linked immunosorbent assays. The survival time of mice was evaluated after challenge with tachyzoites of T. gondii RH strain. Additionally, the number of cysts in the brain was determined after intragastric challenge with cysts of T. gondii PRU strain.

Results: Mice vaccinated with different immunization regimens (peptide, pROM4 and pROM4/peptide) elicited specific humoral and cellular responses, with high levels of IgG, IgG2a, and interferon (IFN)- $\gamma$. Moreover, IgG, IgG2a and IFN- $\gamma$ levels were highest in the pROM4/peptide group. Immunized mice, especially those in the pROM4/ peptide group, had prolonged survival times after challenge with tachyzoites and reduced numbers of brain cysts after infection compared with negative controls.

Conclusion: A DNA prime-peptide boost regimen based on ROM4 elicited the highest level of humoral and cellular immune responses among immunization regimens, and may be a promising approach to increase the efficacy of DNA immunization.

Keywords: Toxoplasma gondii, Vaccine, ROM4, Peptide, Bioinformatics, Immunization strategy

\footnotetext{
* Correspondence: shenyi.he@hotmail.com

${ }^{1}$ Department of Parasitology, Shandong University School of Medicine, Jinan,

Shandong Province 250012, People's Republic of China

Full list of author information is available at the end of the article
} International License (http://creativecommons.org/licenses/by/4.0/), which permits unrestricted use, distribution, and reproduction in any medium, provided you give appropriate credit to the original author(s) and the source, provide a link to the Creative Commons license, and indicate if changes were made. The Creative Commons Public Domain Dedication waiver (http://creativecommons.org/publicdomain/zero/1.0/) applies to the data made available in this article, unless otherwise stated. 


\section{Background}

Toxoplasma gondii (T. gondii) is an obligate intracellular protozoan parasite with an extremely broad host range inclusive of almost all warm-blooded animals, including human [1]. Toxoplasmosis is a zoonotic parasitic disease with a worldwide distribution that poses a serious risk to animal husbandry and human health [2]. T. gondii infection is usually asymptomatic, but not in immunosuppressed patients (e.g. organ transplantation, malignant tumor radiotherapy, and HIV infection). Infection can spread widely, involving the brain, eyes, lymph nodes, can damage multiple organs, and even cause death. T. gondii infection in pregnant women can be transmitted to the fetus via the placenta, leading to miscarriage, premature birth, stillbirth, fetal malformation, and eye toxoplasmosis [3].

There are three infectious stages in the $T$. gondii life cycle: tachyzoites, cysts, and oocysts [4]. Tachyzoites cause acute infection, while chronic infection is mainly mediated by bradyzoites in cysts. When the host immune function is compromised, bradyzoites transform to tachyzoites [3]. Current drugs are effective for the treatment of toxoplasmosis (acute or reactivated) but they cause many side effects, especially in the fetus, and have no effect on cysts and bradyzoites [5]. Therefore, a safe and effective vaccine is important for the prevention and control of toxoplasmosis. DNA vaccines induce a persistent and strongly protective immune response [6, 7]. For example, $T$. gondii surface antigen 1 (SAG1) induces effective and durable humoral and cellular immune responses in immunized mice and is regarded as a standard compared to other antigens [8].

T. gondii expresses several proteases, including serine, cysteine, and aspartic proteases, which play an important role in infection [9-11]. Rhomboid proteases belong to the serine protease family. T. gondii expresses 5 nonmitochondrial rhomboid intramembrane proteases, TgROM1 to TgROM5. ROM proteins affect the cleavage of microneme protein (MIC), which plays an essential role in $T$. gondii invasion $[12,13]$. TgROM4 is expressed primarily in tachyzoites, at lower levels in bradyzoites, and is weakly detected in sporozoites [12, 13]. TgROM4 is localized to the plasma membrane of T. gondii and uniformly distributed on the parasite surface $[12,13]$. When T. gondii effectively invades host cells, TgROM4 plays an important role in regulating microneme protein 2 (MIC2), MIC3, MIC6, and apical membrane antigen 1 (AMA1) [14-16]. Li et al. showed that a TgROM1 DNA vaccine was protective against $T$. gondii in mice [17]. We therefore used bioinformatics to examine whether ROM4 is antigenic. Bioinformatics is a powerful approach for genomics and proteomics and has been widely used to predict and analyze protein antigenic epitopes [18, 19].
Immunization strategy is also a key factor in determining the effectiveness of the immune response and has gained increasing importance in vaccine research $[20,21]$. In particular, a prime-boost vaccination strategy induced a more efficient humoral and cellular immune response [22-24]. Studies have shown that a synthetic multiple antigenic peptides (MAP) vaccine induces protective immunity against intracellular parasites, including $T$. gondii $[25,26]$.

In the present study, we analyzed the ROM4 protein to identify potential antigenic epitopes using a bioinformatics approach. A eukaryotic expression plasmid pEGFP-C1-ROM4 (pROM4) was constructed and used in a DNA prime-peptide boost vaccination regimen to immunize mice and assess protection against $T$. gondii infection. Furthermore, we assessed the protective efficacy of different vaccination strategies (peptide, pROM4, and DNA/peptide).

\section{Methods \\ Prediction of linear-B cell and Th cell epitopes}

The TgROM4 (scaffold no. TGG995368, chromosome VIII) nucleotide (GenBank ID: AY704175.1) and amino acid sequences (GenBank ID: AAU11320.1) were obtained from GenBank. Protein epitopes determine antigen specificity [27, 28]. The linear-B cell epitopes of ROM4 were analyzed using DNASTAR (Madison, WI, USA). The PROTEAN subroutine was used to predict the antigenic index and ROM4 surface probability. Peptides with a good antigenic index and surface probability were chosen. The Immune Epitope Database (IEDB) (http://tools.immuneepitope.org/mhcii) online service was used to analyze the half-maximal inhibitory concentration (IC50) values of peptides that bind to the major histocompatibility complex (MHC) class II molecules of ROM4.

\section{Mice and parasites}

Female BALB/c mice (7-8 weeks old) were purchased from the Shandong University Laboratory Animal Center (Shandong, China). All mice were maintained under specific-pathogen-free conditions. All animal experiments were approved by the Ethics Committee on Animal Experiments of the Medical School of Shandong University.

The $T$. gondii RH strain was used to challenge BALB/c mice. Tachyzoites were extracted from human foreskin fibroblast cells $1 \mathrm{~h}$ before injection to ensure freshness prior to challenge. About $8 \times 10^{9}$ tachyzoites were used to create soluble tachyzoite antigens (STAg) after isolation by centrifugation, and resuspended in sterile PBS as previously described [5], while about $8 \times$ $10^{6}$ tachyzoites were used to extract total RNA with TRIzol Reagent (Life Technologies, Carlsbad, CA, USA). After 
tachyzoites were lysed with $1 \mathrm{ml}$ TRIzol, $0.2 \mathrm{ml}$ chloroform was added, and the homogenate was separated into three layers after centrifugation. RNA was precipitated from the upper aqueous layer with isopropanol and then was washed to remove impurities. RNA was resuspended in RNase-free water and incubated in a water bath at 55$60{ }^{\circ} \mathrm{C}$ for $10-15 \mathrm{~min}$. The RNA was reverse-transcribed into cDNA with RevertAid First Strand cDNA Synthesis Kit according to the manufacturer's protocol (Thermo Scientific, Carlsbad, CA, USA)

\section{Eukaryotic expression plasmid construction and preparation}

The whole SAG1 open reading frame (ORF) was amplified by polymerase chain reaction (PCR) from $T$. gondii tachyzoite cDNA (forward primer: 5'-CCGCTCGAG CTATGTCGGTTTCGCTGCACCAC -3', reverse primer: 5' -CGGAATTCTCACGCGACACAAGCTGCGAT3'). XhoI and EcoRI restriction sites are underlined, respectively. The ROM4 ORF was amplified by PCR from T. gondii tachyzoite cDNA (forward primer: 5'-CCG CTCGAGTGGCGTCCCCTCACGGATCC-3', reverse primer: 5'-GGGGTACCTTACGGTTCAAGGTAATAC TGCGC-3'). XhoI and KpnI restriction sites are underlined, respectively.

The SAG1 and ROM4 DNA fragments were respectively inserted into a pEASY-T1 vector (TransGen Biotech, Beijing, China). After sequencing, SAG1 and ROM4 were respectively subcloned into the eukaryotic expression plasmid pEGFP-C1 (Novagen, Billerica, MA, USA) to form pEGFP-C1-SAG1(pSAG1) and pEGFPC1-ROM4 (pROM4), respectively using the XhoI, EcoRI and $X h o I, K p n I$ restriction sites. The new recombinant plasmids were then used to transfect HEK 293-T cells.

Recombinant pSAG1 and pROM4 were transformed into Escherichia coli DH5 . After being verified by PCR, double restriction enzyme digestion and double stranded sequencing, recombinant plasmids were extracted using an endotoxin-free mega kit following the manufacturer's instructions (Qiagen, Hilden, Germany), and stored at $-20{ }^{\circ} \mathrm{C}$ until use. pSAG1 and pROM4 concentrations were determined by A260/A280 measurement.

\section{Preparation of polypeptide}

ROM4 peptide 405-424 (YALLGALIPYCVEYWKSIPR) was synthesized by SynPeptide Co Ltd (Shanghai, China), and purity was confirmed by analytic HPLC.

\section{Expression of pEGFP-C1-ROM4 in HEK 293-T cells}

HEK 293-T cells were maintained in Dulbecco's Modified Eagle Medium (DMEM) supplemented with streptomycin $(100 \mathrm{mg} / \mathrm{ml})$, penicillin $(100 \mathrm{IU} / \mathrm{ml})$ and $10 \%$ fetal bovine serum (FBS), at $37{ }^{\circ} \mathrm{C}$ in a humidified atmosphere with 5\% CO2. Before transfection, HEK293 cells (about $1-2 \times 10^{5} /$ well) were transferred into Costar 6-well culture plates (Sigma-Aldrich, St. Louis, MO, USA). When HEK 293-T density reached 80-90\%, pEGFP-C1, pSAG1 and pROM4 were transfected with Lipofectamine 2000 reagent (Invitrogen, Carlsbad, CA, USA) according to the manufacturer's protocol. Plasmids (2.5 $\mathrm{\mu g} /$ well) were mixed with Lipofectamine 2000 reagent $(7 \mu \mathrm{L} /$ well $)$ in DMEM, incubated at room temperature for $20 \mathrm{~min}$, and then added drop by drop on to HEK 293-T cells. After incubation for $6 \mathrm{~h}$, the medium was exchanged with medium containing $10 \%$ FBS. After $48 \mathrm{~h}$, the cells from different groups (control, pEGFP-C1, pSAG1 and pROM4) were observed using fluorescence microscopy under a blue laser.

\section{Immunization and challenge}

All BALB/c mice were divided randomly into six groups (27 per group). Mice were immunized twice at 2-week intervals with PBS $(100 \mu \mathrm{l})$, pEGFP-C1 $(100 \mu \mathrm{g}), \mathrm{pSAG} 1$ $(100 \mu \mathrm{g})$, pROM4 $(100 \mu \mathrm{g})$, peptide $(100 \mu \mathrm{g})$, or pROM4 $(100 \mu \mathrm{g}) /$ peptide $(100 \mu \mathrm{g})$ by intramuscular injection. All groups were immunized four times. The last group was injected with pROM4 the first two times and with peptide the last two times.

Two weeks after the final immunization, seven mice per group were euthanized and splenocytes harvested under aseptic conditions for cytokine detection. Ten mice from each group were challenged intraperitoneally with $T$. gondii $\mathrm{RH}$ strain $\left(1 \times 10^{4}\right.$ tachyzoites $)$. Changes in health status were observed and survival times recorded. The remaining mice were infected intragastrically with 20 cysts of $T$. gondii PRU strain. At 1 month after challenge, brains were removed and homogenized in $1 \mathrm{ml}$ PBS. The number of cysts in each brain was determined by counting three samples of $10 \mu \mathrm{l}$ from the homogenate via an optical microscope, and the average value was used to evaluate the effect of the vaccine.

\section{Determination of antibodies}

Serum samples were collected from all mice prior to each immunization and 2 weeks after the final injection. Anti-T. gondii IgG, IgG1, and IgG2a antibodies were detected using enzyme-linked immunosorbent assay (ELISA). Briefly, 96-well plates (Costar) were coated with STAg $(10 \mu \mathrm{g} /$ well $)$ and incubated at $4{ }^{\circ} \mathrm{C}$ overnight. Plates were washed three times with special ELISA solution and blocked with PBS containing 1\% BSA for $2 \mathrm{~h}$ at room temperature, followed by three washes. Plates were then incubated with PBS-diluted mouse sera for $1 \mathrm{~h}$ at $37{ }^{\circ} \mathrm{C}$. After washing, plates were incubated with horseradish peroxidase (HRP)-conjugated anti-mouse IgG (diluted 1:4000 in PBS-1\% BSA), IgG1 (1:2000), and IgG2a (1:2000) for $1 \mathrm{~h}$ at $37{ }^{\circ} \mathrm{C}$, washed with ELISA 
solution, with orthophenylene diamine (Sigma) and $0.15 \% \mathrm{H}_{2} \mathrm{O}_{2}$ added. Plates were then incubated in the dark for $30 \mathrm{~min}$ at $37{ }^{\circ} \mathrm{C}$, and the reaction stopped by adding $2 \mathrm{M} \mathrm{H}_{2} \mathrm{SO}_{4}$. The optical density was measured at $490 \mathrm{~nm}$ using an ELX800 ELISA reader (BioTek, Winooski, VT, USA). All samples were run four times.

\section{Cytokine assays}

Spleens were isolated from seven mice per group 2 weeks after the last immunization and used to form a cell suspension adjusted to $1 \times 10^{6}$ cells $/ \mathrm{ml}$. Then, $1 \times 10^{5}$ spleen cells was added to each well in a 96-well plate with $10 \mu \mathrm{l}$ non-specific irritant Con A $(5 \mu \mathrm{g} / \mathrm{ml})$ and cultured at $37{ }^{\circ} \mathrm{C}$ in $5 \% \mathrm{CO} 2$. Cell-free supernatants were harvested and assayed for interleukin-2 (IL-2) and IL-4 at $24 \mathrm{~h}$, IL10 at $72 \mathrm{~h}$, and IL-12 (p70) and interferon (IFN)- $\gamma$ at $96 \mathrm{~h}$. The concentrations of cytokines were measured by ELISA according to the manufacturer's instructions (R\&D Systems, Minneapolis, MN, USA). The detection limits of the assays for IFN- $\gamma$, IL-2, IL-4, IL-10 and IL-12 (p70) were $2.1 \mathrm{pg} / \mathrm{mL}, 3.5 \mathrm{pg} / \mathrm{mL}, 2.3 \mathrm{pg} / \mathrm{mL}, 1.97 \mathrm{pg} / \mathrm{mL}$ and $2.24 \mathrm{pg} / \mathrm{mL}$, respectively. All samples were run four times.

\section{Statistical analysis}

SPSS 17.0 (IBM, Chicago, IL, USA) was used for statistical analysis. The mean of total IgG, IgG1, IgG2a, cytokine levels and cyst numbers among the different groups were analyzed and determined by one-way analysis of variance (ANOVA). Mouse survival time was compared using the Kaplan-Meier method. When a significant difference $(P<0.05)$ was observed among treatments, a Tukey's studentized range test was used for post-test comparisons.

\section{Results \\ Epitope analysis}

DNASTAR was used to determine hydrophilicity plots, flexible regions, antigenic index, and surface probability. SAG1 had an excellent antigenic index and surface probability, making it a good vaccine candidate (Fig. 1). Prediction results indicated that ROM4 had a better antigenic index than SAG1. Moreover, ROM4 had significantly more surface probability and more flexible regions than SAG1.

The ROM4 Th predicted epitopes, and the IC50 values of ROM4 peptides binding to MHC class II molecules, and the minimum percentile ranks of each ROM4 MHC II allele are listed in Table 1 . The minimum percentile ranks of HLA-DRB1*01:01, H2-IAb, H2-IAd, and H2IEd alleles of ROM4 were smaller than those of SAG1, indicating that ROM4 may have better Th epitopes than SAG1.

\section{Identification and expression of recombinant plasmid}

In pSAG1-, pROM4- and vector pEGFP-C1-transfected cells, proteins emitted green fluorescence upon exposure to a blue laser when observed by fluorescence microscopy, whereas no fluorescence was observed in control cells.

\section{Detection of antibody responses in immunized mice}

The levels of $T$. gondii-specific IgG antibodies in immunized mice were determined by ELISA at weeks 0 , 2, 4, 6, and 8. Elevated IgG levels were detected in the sera of mice immunized with pSAG1, peptide, pROM4, or pROM4/peptide, compared to negative controls (PBS or pEGFP-C1) $(P<0.05)$ (Fig. 2). Importantly, $T$. gondii-specific IgG antibodies were not detected in the sera of mice injected with PBS or pEGFP-C1. Furthermore, mice immunized by pROM4/ peptide generated the highest levels of $T$. gondii-specific IgG antibodies among all group (respectively increased by $24 \%, 38 \%$, and $41 \%$ compared with pSAG1, pROM4, or peptide immunization, $P<0.05)$. No statistical differences were found between PBS and pEGFP-C1, pROM4 and peptide $(P>0.05)$.

The levels of IgG subclass (IgG1 and IgG2a) at 2 weeks after the last injection were assayed to determine whether a Th1 and/or Th2 response was elicited (Fig. 3). An apparent predominance of IgG2a over IgG1 was detected in immunized mice, suggesting a shift toward a Th1 type response. Furthermore, IgG2a levels in mice immunized with $\mathrm{pROM} 4 /$ peptide respectively increased by about 2 -fold compared to negative controls and $16 \%$, $31 \%$, 36\% compared to mice injected with pSAG1, pROM4 or peptide $(P<0.05)$. IgG2a levels in mice immunized with pSAG1 were increased by $10.3 \%$ compared with mice injected with pROM4, but with no statistical difference $(P>0.05)$. There was no statistical difference in IgG2a levels between the pROM4 and peptide groups $(P>0.05)$.

\section{Cytokine production}

Culture supernatants of immunized splenocytes were obtained 2 weeks after the last injection and IFN- $\gamma$, IL-2, IL-12(p70), IL-4 and IL-10 activity determined. IFN- $\gamma$ levels in mice immunized with pSAG1, pROM4, peptide or pROM4/peptide were increased about 12-fold, 10-fold, 9-fold, and 15-fold $(P<0.05)$, respectively, compared to PBS injected groups (Table 2). Moreover, mice immunized with pROM4/peptide generated higher IFN- $\gamma$ levels than mice injected with pSAG1, peptide or pROM4 $(P<0.05)$. IFN- $\gamma$ levels in mice immunized with pSAG1 were increased by about $15 \%$ compared with pROM4, but there was no statistical difference. No difference in IFN- $\gamma$ levels was found between the PBS and pEGFP-C1 groups. Significantly 


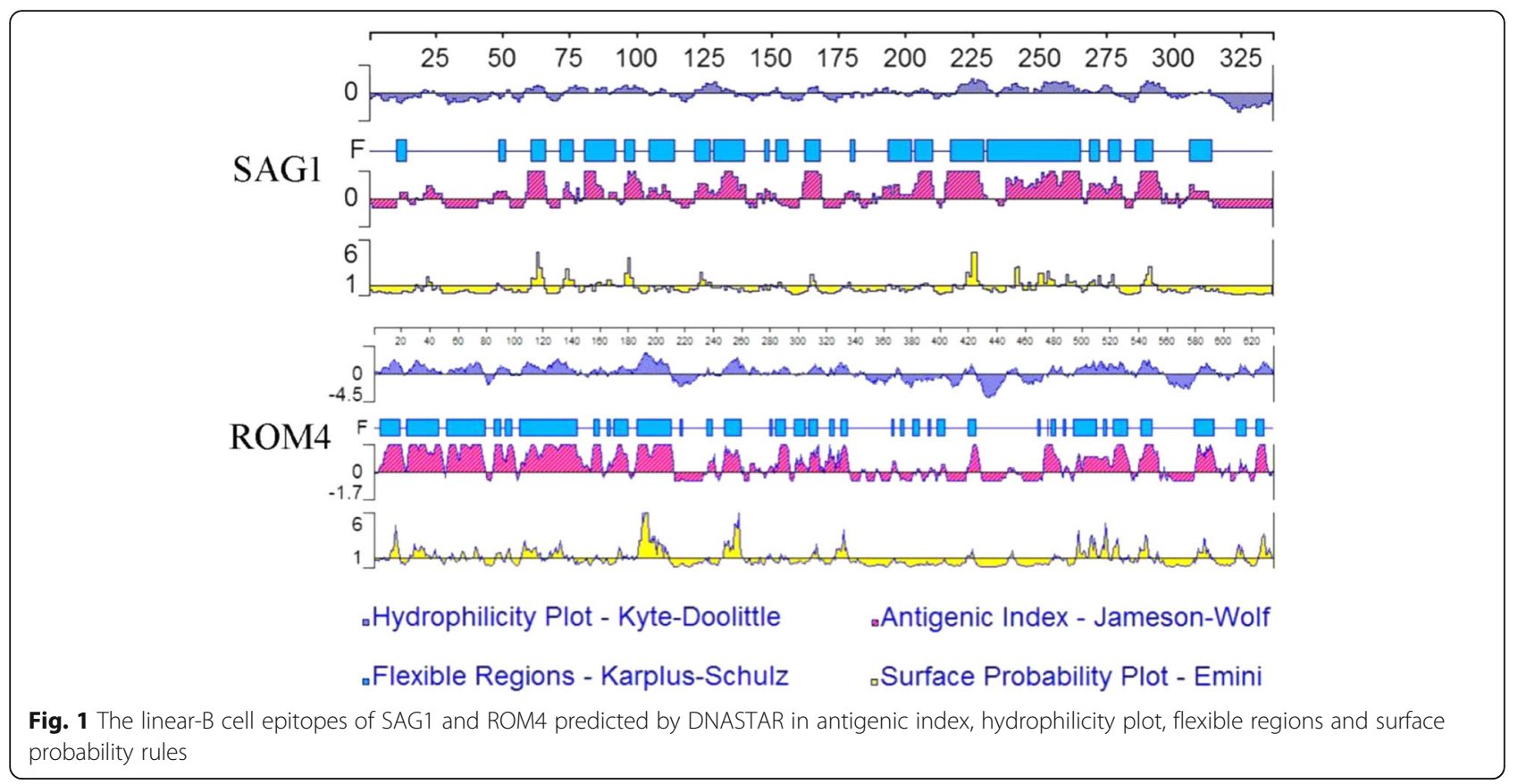

higher levels of IL-2 and IL-12(p70) were generated from mice vaccinated with pSAG1, peptide, pROM4 or pROM4/peptide, compared to mice immunized with PBS or an empty plasmid $(P<0.05)$. IL-2 levels in $\mathrm{pROM} 4 /$ peptide immunized mice increased by $38 \%, 62 \%$, and $63 \%$, compared to pSAG1, pROM 4 or peptide groups $(P<0.05)$, respectively, and the highest IL-12 level also was detected in the pROM4/peptide group $(P<0.05)$ (Table 2$)$. The highest level of IL-4 or IL-10 was detected in mice immunized with pROM4/

Table 1 IC50 values for SAG1 and ROM4 peptide binding to $\mathrm{MHC}$ class II molecules obtained using the immune epitope database $^{a}$

\begin{tabular}{|c|c|c|c|c|}
\hline \multirow[t]{2}{*}{ MHC II Allele } & \multicolumn{2}{|c|}{ Start-Stop ${ }^{c}$} & \multicolumn{2}{|c|}{ Percentile Rank ${ }^{d}$} \\
\hline & $\overline{S A G 1}$ & ROM4 & $\overline{S A G 1}$ & ROM4 \\
\hline \multirow[t]{2}{*}{ HLA-DRB1*01:01 } & $12-26$ & $570-584$ & 0.88 & 0.09 \\
\hline & $35-49$ & $399-413$ & 2.74 & 0.6 \\
\hline \multirow[t]{2}{*}{$\mathrm{H} 2-\mathrm{IAb}$} & $26-40$ & $483-497$ & 2.15 & 1.75 \\
\hline & $297-313$ & $42-56$ & 2.81 & 1.95 \\
\hline \multirow[t]{2}{*}{$\mathrm{H} 2-\mathrm{IAd}$} & $21-35$ & $502-516$ & 0.34 & 0.39 \\
\hline & 168-182 & $529-543$ & 1.22 & 0.58 \\
\hline \multirow[t]{2}{*}{$\mathrm{H} 2-\mathrm{IEd}$} & $14-28$ & 179-193 & 18.45 & 4.91 \\
\hline & $34-48$ & $412-426$ & 30.62 & 11.21 \\
\hline
\end{tabular}

${ }^{a}$ The immune epitope database (http://tools.immuneepitope.org/mhcii). The prediction was run for three times

${ }^{\mathrm{b}} \mathrm{H} 2-\mathrm{IAb}, \mathrm{H} 2$-IAd and H2-IEd alleles are mouse MHC class II molecules; the HLA-DRB1*01:01 allele is a human MHC class II molecule

'We chose 15 amino acids for analysis each time

${ }^{\mathrm{d}}$ Low percentile $=$ high level binding, high percentile = low level binding peptide, but there was no statistically significant difference between pROM4/peptide and other groups $(P>0.05)$.

\section{Protection of DNA vaccine against $T$. gondii}

Survival days after challenge by $1 \times 10^{4}$ tachyzoites of T. gondii RH strain were tabulated (Fig. 4). Mice injected with PBS or empty vector developed some symptoms characterized by reduced feeding, arched back, vertical hair and feces around the anus beginning at 2 days. Mice also started to die at 3 days after tachyzoite challenge, and all mice in negative controls were dead at day 6 postinfection. Mice showed symptoms at later timepoints and survived longer following single or multiple-immunization compared with the PBS or pEGFP-C1 groups $(P<0.05)$. Moreover, mice immunized with pROM4/peptide exhibited the latest onset of symptoms (beginning at 9 days after challenge) and the longest survival time (18 days) among all groups $(P<0.05)$.

Two weeks after the last injection, all the mice were challenged intragastrically with 20 cysts from the $T$. gondii PUR strain to evaluate the protective effect of the DNA vaccine. Brain cysts were reduced in mice vaccinated with pSAG1, pROM4, peptide, or pROM4/peptide compared to mice injected with PBS or pEGFP-C1 $(P<0.05)$ (Table 3$)$. The cyst numbers were reduced to $57 \%$ of controls in the brains of mice immunized with $\mathrm{pROM} 4 /$ peptide. However, no statistically significant difference was found between the pSAG1, pROM4 and peptide groups $(P>0.05)$, or between the PBS and pEGFP-C1 groups $(P>0.05)$. 


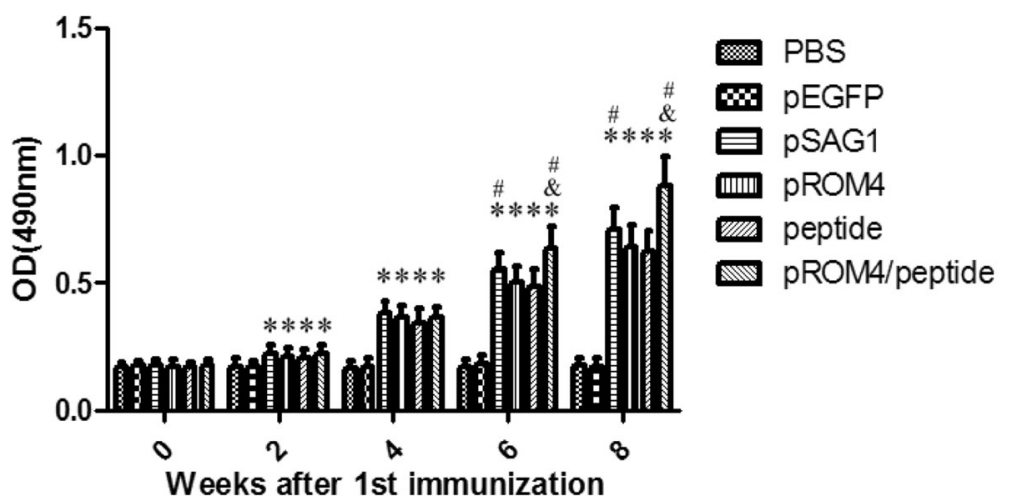

Fig. 2 Detection of specific lgG antibodies in sera of vaccinated mice. Sera was collected 2 days before each immunization and determined using ELISA. All samples were performed four times. The results are mean of 27 mice per group and expressed as the mean of the optical density of $490 \pm$ SD. ${ }^{*} P<0.05$, as compared with PBS and pEGFP-C1; \# $P<0.05$, as compared with ROM4 or peptide; \& $P<0.05$, as compared with pSAG1

\section{Discussion}

In the present study, ROM4 B-cell and T-cell epitopes were analyzed using DNAStar software and online services. This approach yielded several potential ROM4 T-cell epitopes, suggesting that it may serve as a vaccine against T. gondii. Following bioinformatics analysis, the ROM4 gene was cloned into the eukaryotic expression plasmid pEGFP-C1, and recombinant plasmids transfected into HEK 293-T cells. The results showed that the recombinant plasmid pROM4 was efficiently transcribed and expressed in eukaryotic cells.

Cellular immunity mediated by $\mathrm{T}$ cells plays an important role in T. gondii infection [29]. To develop an effective vaccine against toxoplasmosis, it is necessary to elucidate which type of Th cell-mediated immune response is elicited. Several studies have indicated that CD4+ and CD8+ $\mathrm{T}$ cells play a major role in the anti- $T$. gondii response [30, 31]. Cytotoxic CD8+ T cells and the Th1 cytokine IFN- $\gamma$ play an important role in $T$. gondii immunity [30, 32]. CD4 + T lymphocytes are divided into two subtypes, Th1 and Th2, based on the cytokines produced post-stimulation. Th1 cells produce IL-2, IL-12 and IFN- $\gamma$, and Th2 cells secrete IL-4, IL5 , IL-6, and IL-10 [32]. IFN- $\gamma$ plays a leading role in restricting both acute phase tachyzoite breeding and chronic phase bradyzoite activation in the cysts. Studies have shown that good DNA vaccines tend to stimulate a Th1-type rather than a Th2-type immune response $[8,33]$. The Th2 cytokines IL- 4 and IL-10 limit the differentiation of $\mathrm{CD} 4+\mathrm{T}$ cell to $\mathrm{Th} 1$, thereby decreasing the level of IFN- $\gamma[34,35]$. In addition, B lymphocytes play an important role in anti-infection immunity by producing IgG anti- $T$. gondii antibodies, and inhibiting parasite and host cell adhesion, especially during vaccine-induced protective immunity [36, 37].

In the current study, IgG antibody levels in immunized mice were significantly higher than in negative controls, illustrating that the vaccine constructed here induces a sufficient amount of $T$. gondii-specific IgG antibodies.

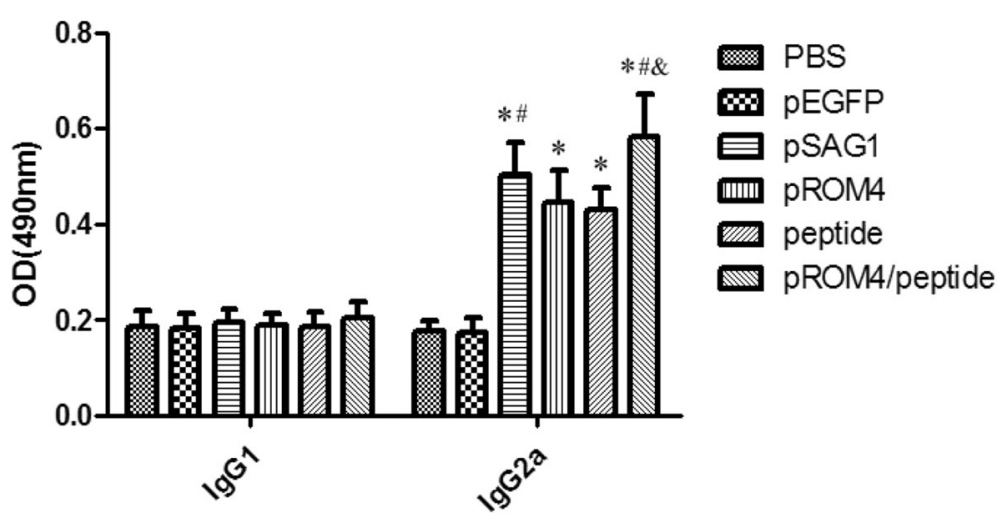

Fig. 3 Detection of IgG1 and IgG2a levels in immunized mice sera by ELISA. Sera was collected at 2 weeks after the last injection and detected by ELISA. All samples were performed four times. The results are mean of 27 mice per group and expressed as the mean of the optical density of $490 \pm$ SD. ${ }^{*} P<0.05$, as compared with PBS and pEGFP-C1; \# $P<0.05$, as compared with ROM4 or peptide; \& $P<0.05$, as compared with pSAG1 
Table 2 Cytokine production by splenocyte ${ }^{\mathrm{a}}$ cultures from immunized BALB/c mice

\begin{tabular}{|c|c|c|c|}
\hline \multirow[t]{2}{*}{ Group } & \multicolumn{3}{|c|}{ Cytokine production $(\mathrm{pg} / \mathrm{mL})^{\mathrm{b}}$} \\
\hline & IFN- $\gamma$ & IL-2 & IL-12 \\
\hline PBS & $48.9 \pm 6.5$ & $33.2 \pm 3.56$ & $37.5 \pm 7.3$ \\
\hline pEGFP-C1 & $51.1 \pm 7.1$ & $33.9 \pm 5.3$ & $38.1 \pm 6.0$ \\
\hline pSAG1 & $613.9 \pm 59.3^{*}$ & $247.9 \pm 29.0^{*}$ & $160.2 \pm 35.5^{*}$ \\
\hline pROM4 & $531.8 \pm 63.3^{*}$ & $212.4 \pm 22.4^{*}$ & $146.1 \pm 31.1^{*}$ \\
\hline peptide & $488.6 \pm 79.1^{*}$ & $210.1 \pm 20.7^{*}$ & $132.9 \pm 26.0^{*}$ \\
\hline pROM4/peptide & $778.2 \pm 93.2^{* \# \&}$ & $343.2 \pm 22.4^{* \# \&}$ & $247.1 \pm 48.8^{* \# \&}$ \\
\hline \multirow[t]{2}{*}{ Group } & \multicolumn{3}{|c|}{ Cytokine production $(\mathrm{pg} / \mathrm{mL})^{b}$} \\
\hline & IL-4 & $\mid \mathrm{IL}-10$ & \\
\hline PBS & $38.9 \pm 8.9$ & $39.5 \pm 8.5$ & \\
\hline pEGFP-C1 & $38.6 \pm 10.7$ & $41.5 \pm 6.8$ & \\
\hline pSAG1 & $36.5 \pm 4.0$ & $39.7 \pm 5.9$ & \\
\hline pROM4 & $40.2 \pm 5.8$ & $40.7 \pm 4.8$ & \\
\hline peptide & $38.1 \pm 7.7$ & $37.6 \pm 8.6$ & \\
\hline pROM4/peptide & $41.2 \pm 5.7$ & $42.4 \pm 6.5$ & \\
\hline
\end{tabular}

${ }^{a}$ Splenocytes from seven mice per group 2 weeks after the final immunization. All samples were performed four times

${ }^{b}$ Values for IL-12 (p70) and IFN- $\gamma$ at $96 \mathrm{~h}, \mathrm{IL}-2$ and IL-4 at $24 \mathrm{~h}, \mathrm{LL}-10$ at $72 \mathrm{~h}$ are expressed as mean \pm SD

${ }^{*} P<0.05$, as compared with PBS and pEGFP-C $1 ;{ }^{\#} P<0.05$, as compared with pROM4 or peptide; ${ }^{\&} P<0.05$, as compared with $\mathrm{PSAG} 1$
IgG, IgG2a, IL-2, IL-12 and IFN- $\gamma$ levels in immunized mice were higher than in negative controls, and IgG1, IL-4, and IL-10 levels were similar between all groups. These results suggest that pSAG1, pROM4 and peptide mainly induced a Th1 immune response. In addition, IgG and IgG2a levels in pSAG1 immunized mice were higher than in pROM4 immunized mice. This is not in accord with the results of the bioinformatics analysis, suggesting that bioinformatics analysis is predictive and that the predicted protein antigenic epitopes need to be verified by an experimental approach.

To assess the protective efficacy of the vaccine, BALB/ c mice were infected with $T$. gondii tachyzoites and cysts. The results showed that the onset of symptoms were later in immunized mice, which had prolonged survival times and reduced brain cysts compared with negative controls. Zhang et al. [38] constructed a pVAX-TgROM4 DNA vaccine and evaluated the immunogenicity in Kunming mice. They showed that the TgROM4 DNA vaccine induced strong humoral and cellular responses and was a potential vaccine candidate against toxoplasmosis. Our study confirmed the protective immunity of ROM4 DNA vaccine against toxoplasmosis in BALB/C mice. However, we added the ROM4/peptide group and showed that the group had the greatest protective effect. Of note, some parameters (the parasite strain, vaccine constructs, dose of vaccination, mouse strain, et al.) might affect the assessment of protective immunity in different studies [6].

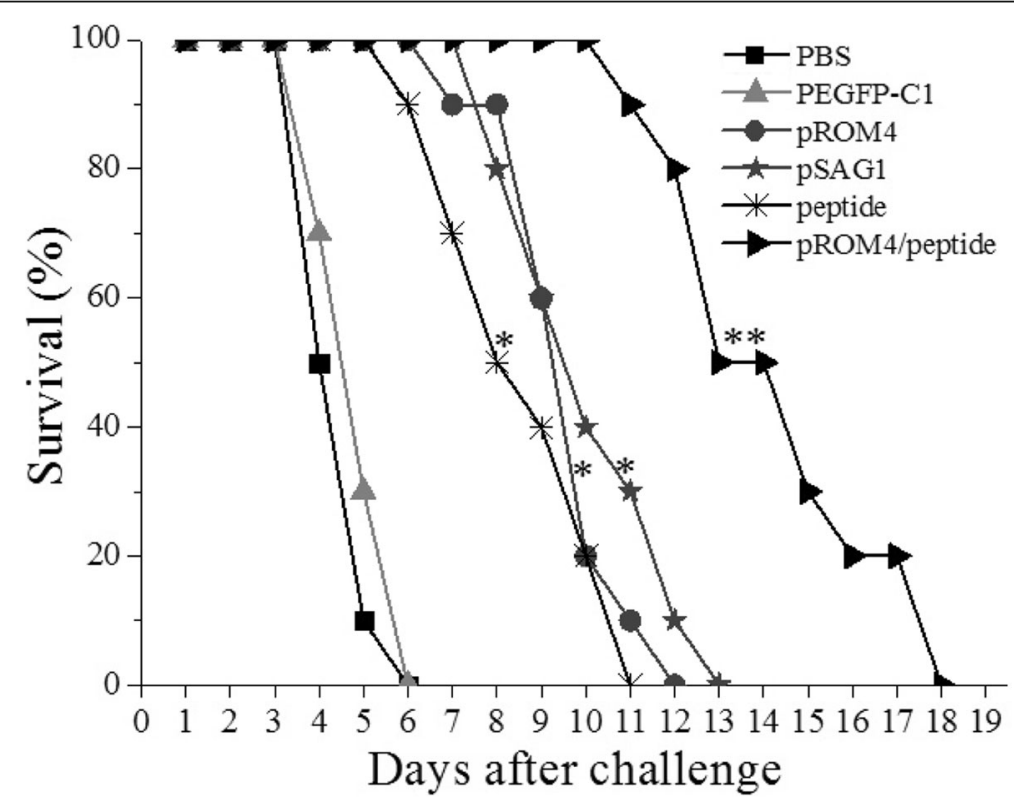

Fig. 4 Survival curves of injected BALB/C mice against T. gondii challenge. The six groups of mice were challenged with $1 \times 10^{4}$ tachyzoites of virulent $T$. gondii RH strain 2 weeks after the last immunization. Each group was composed of ten mice and survival time was monitored daily for 18 days after challenge. ${ }^{*} P<0.05$, as compared with PBS or pEGFP-C1; ${ }^{* *} P<0.05$, as compared with pSAG1, pROM4 or peptide 
Table 3 Brain cyst burden in injected mice after infection with cyst of PRU strain

\begin{tabular}{ll}
\hline Challenged group $^{a}$ & Brain cysts per mouse (mean \pm SD) \\
\hline PBS & $1283 \pm 193$ \\
pEGFP-C1 & $1251 \pm 199$ \\
pSAG1 & $812 \pm 92^{*}$ \\
pROM4 & $761 \pm 102^{*}$ \\
peptide & $827 \pm 100^{*}$ \\
pROM4/peptide & $551 \pm 89^{* \# \&}$
\end{tabular}

${ }^{a}$ Ten mice from each group were challenged intragastrically by 20 cysts 2 weeks after the last immunization

${ }^{b}$ The mean number of cysts of each group was obtained from every mice brain cysts in the group

${ }^{*} P<0.05$, as compared with PBS or pEGFP-C1; ${ }^{*} P<0.05$, as compared with ROM4 or peptide; ${ }^{\&} P<0.05$, as compared with $\mathrm{pSAG} 1$. All samples were performed four times

DNA vaccines often induce insufficient protective immunity against $T$. gondii challenge [39]. Prime-boost vaccination strategies have been used to enhance immune responses of some DNA vaccines [5, 22]. The first immunization primes the immune response and subsequent immunizations trigger the further expansion of antigen specific cells and selection of cells with high antigen avidity [40]. Synthetic MAP contains a high concentration of the relevant antigen to induce immune responses to predefined epitopes and leads to protective immunity [25, 41]. Meng et al. [5] used the DNA prime-peptide boost vaccination regimen to enhance the effectiveness of $T$. gondii DNA vaccines, which produced a stronger immune response and better protection compared to priming with polypeptide and boosting with DNA.

The prime-boost vaccination usually include two strategies: homologous boosting and heterologous boosting. Researches display that homologous boosting is relatively inefficient at boosting cellular immunity than that of heterologous boosting,. So-called heterologous boosting means that different antigen-delivery systems are used [42]. In the present study, we selected a polypeptide and immunized mice using a DNA-priming and polypeptide-boosting regimen. The results showed that the levels of IgG, IgG2a over IgG1, IL-2, IL-12 and IFN$\gamma$ in the DNA/peptide group were highest. In addition, mice treated with the DNA/peptide exhibited the longest survival time after challenge with tachyzoites and the greatest reduction in brain cysts after infection by cysts.

\section{Conclusion}

In conclusion, T. gondii ROM4 is a potential DNA vaccine candidate against toxoplasmosis. A DNA primepeptide boost regimen based on ROM4 elicited the highest level of humoral and cellular immune responses among the immunization regimens, indicating it may be a promising approach to generate an efficient protective immune response and prevent $T$. gondii infection.

\section{Acknowledgments}

This work was supported, in part, by grants from the National Natural Science Foundation of China (Grant Nos. 81071373 and 81271857), the State Key Laboratory of Veterinary Etiological Biology (Grant No. SKLVEB2011KFKT005) and the Shandong Provincial Natural Science Foundation (Grant No. ZR2009CM079).

\section{Availability of data and materials}

TgROM4 nucleotide (GenBank ID: AY704175.1) and amino acid sequences (GenBank ID: AAU11320.1).TgSAG1 nucleotide (GenBank ID: JX045360.1) and amino acid sequences (GenBank ID: AFO54847.1).

\section{Authors' contributions}

$\mathrm{YH}$ carried out the experiments and drafted the manuscript. $A Z, G L$ and GZ revised the manuscript. LW, JG, PS, JZ, HZ and $H C$ conducted the experiments and revised the manuscript. SH conceived and designed the study and revised the manuscript. All of the authors have read and approved the final manuscript.

\section{Competing interests}

The authors declare that they have no competing interests.

\section{Consent for publication}

The manuscript is approved by all authors for publication. We would like to declare on behalf of my co-authors that the work described was original research that has not been published previously, and not under.

\section{Ethics approval}

The Institutional Animal Care and Use Committee of Shandong University approved all experimental protocols. Experiments were performed in accordance with the criteria for the care and use of laboratory animals. All mice were maintained in specific pathogen-free conditions, and all efforts were made to minimize suffering. Humane endpoints to reduce pain or distress were used via euthanasia. Mice were sacrificed immediately using $\mathrm{CO}_{2}$ gas before brains were removed. Generally, mice were placed in a chamber and $\mathrm{CO}_{2}$ administered at a concentration of $60-70 \%$ over a 5 -min exposure time, after which the cervical dislocation method was sometimes used.

\section{Author details}

${ }^{1}$ Department of Parasitology, Shandong University School of Medicine, Jinan, Shandong Province 250012, People's Republic of China. Department of Pediatrics, Provincial Hospital Affiliated to Shandong University, Shandong University School of Medicine, 250021 Jinan, Shandong Province, People's Republic of China. ${ }^{3}$ Qilu Hospital of shandong University, Qingdao 266035, Shandong Province, People's Republic of China. ${ }^{4}$ Department of Ji Nan Children's Hospital, 250022 Jinan, Shandong Province, People's Republic of China.

Received: 9 April 2016 Accepted: 10 December 2016

Published online: 11 January 2017

\section{References}

1. Dubey JP. The history of Toxoplasma gondii-the first 100 years. J Eukaryot Microbiol. 2008;55(6):467-75.

2. Cenci-Goga BT, Rossitto PV, Sechi P, McCrindle CM, Cullor JS. Toxoplasma in animals, food, and humans: an old parasite of new concern. Foodborne Pathog Dis. 2011;8(7):751-62.

3. Montoya JG, Liesenfeld O. Toxoplasmosis. Lancet. 2004;363(9425):1965-76. 3.

4. Dubey JP, Lindsay DS, Speer CA. Structures of Toxoplasma gondii tachyzoites, bradyzoites, and sporozoites and biology and development of tissue cysts. Clin Microbiol Rev. 1998;11(2):267-99.

5. Meng M, Zhou A, Lu G, Wang L, Zhao G, Han Y, et al. DNA prime and peptide boost immunization protocol encoding the Toxoplasma gondii GRA4 induces strong protective immunity in BALB/c mice. BMC Infect Dis. 2013;13:494. 5.

6. Jongert E, Roberts CW, Gargano N, Förster-Waldl E, Petersen E. Vaccines against Toxoplasma gondii:challenges and opportunities. Mem Inst Oswaldo Cruz. 2009;104(2):252-66.

7. Hiszczyńska-Sawicka E, Holec-Gasior L, Kur J. DNA vaccines and recombinant antigens in prevention of Toxoplasma gondii infections-current status of the studies. Wiad Parazytol. 2009;55(2):125-39. 
8. Liu KY, Zhang DB, Wei QK, Li J, Li GP, Yu JZ. Biological role of surface Toxoplasma gondii antigen in development of vaccine. World J Gastroenterol. 2006;12(15):2363-8.

9. Kim K. Role of proteases in host cell invasion by Toxoplasma gondii and other Apicomplexa. Acta Trop. 2004;91(1):69-81.

10. Dou Z, Carruthers VB. Cathepsin proteases in Toxoplasma gondii. Adv Exp Med Biol. 2011;2011(712):49-61.

11. Zhao G, Zhou A, Lv G, Meng M, Sun M, Bai Y, et al. Toxoplasma gondii cathepsin proteases are undeveloped prominent vaccine antigens against toxoplasmosis. BMC Infect Dis. 2013;13:207.

12. Dowse TJ, Pascall JC, Brown KD, Soldati D. Apicomplexan rhomboids have a potential role in microneme protein cleavage during host cell invasion. Int J Parasitol. 2005;35(7):747-56.

13. Brossier F, Jewett TJ, Sibley LD, Urban S. A spatially localized rhomboid protease cleaves cell surface adhesins essential for invasion by Toxoplasma. Proc Natl Acad Sci U S A. 2005;102(11):4146-51.

14. Buguliskis JS, Brossier F, Shuman J, Sibley LD. Rhomboid 4 (ROM4) affects the processing of surface adhesins and facilitates host cell invasion by Toxoplasma gondii. PLoS Pathog. 2010;6(4):e1000858.

15. Shen B, Buguliskis JS, Lee TD, Sibley LD. Functional analysis of rhomboid proteases during Toxoplasma invasion. MBio. 2014;5(5):e01795-14.

16. Rugarabamu G, Marq JB, Guérin A, Lebrun M, Soldati-Favre D. Distinct contribution of Toxoplasma gondii rhomboid proteases 4 and 5 to micronemal protein protease 1 activity during invasion. Mol Microbiol. 2015:97(2):244-62.

17. Li J, Han Q, Gong P, Yang T, Ren B, Li S, Zhang X. Toxoplasma gondii rhomboid protein 1 (TgROM1) is a potential vaccine candidate against toxoplasmosis. Vet Parasitol. 2012;184(2-4):154-60.

18. Sollner J, Grohmann R, Rapberger R, Perco P, Lukas A, Mayer B. Analysis and prediction of protective continuous B-cell epitopes on pathogen proteins. Immunome Res. 2008:4:1.

19. Zhang ZW, Zhang YG, Wang YL, Pan L, Fang YZ, Jiang ST, et al. Screening and identification of $B$ cell epitopes of structural proteins of foot-andmouth disease virus serotype Asia I. Vet Microbiol. 2010;140(1-2):25-33.

20. Shedlock DJ, Weiner DB. DNA vaccination: antigen presentation and the induction of immunity. J Leukoc Biol. 2000;68(6):793-806.

21. Liu Q, Singla LD, Zhou H. Vaccines against Toxoplasma gondii: status, challenges and future directions. Hum Vaccin Immunother. 2012;8(9):1305-8.

22. Alekseeva E, Sominskaya I, Skrastina D, Egorova I, Starodubova E, Kushners E, et al. Enhancement of the expression of HCV core gene does not enhance core-specific immune response in DNA immunization: advantages of the heterologous DNA prime, protein boost immunization regimen. Genet Vaccines Ther. 2009;7:7.

23. Zhang $\mathrm{G}$, Huong VT, Battur B, Zhou J, Zhang H, Liao M, et al. A heterologous prime-boost vaccination regime using DNA and a vaccinia virus, both expressing GRA4, induced protective immunity against Toxoplasma gondii infection in mice. Parasitology. 2007;134(Pt 10):1339-46.

24. Min J, Qu D, Li C, Song X, Zhao Q, Li XA, et al. Enhancement of protective immune responses induced by Toxoplasma gondii dense granule antigen 7 (GRA7) against toxoplasmosis in mice using a prime-boost vaccination strategy. Vaccine. 2012;30(38):5631-6.

25. Wang $Y$, Wang $M$, Wang G, Pang A, Fu B, Yin H, et al. Increased survival time in mice vaccinated with a branched lysine multiple antigenic peptide containing B- and T-cell epitopes from T. gondii antigens. Vaccine 2011;29(47):8619-23.

26. Mahajan B, Berzofsky JA, Boykins RA, Majam V, Zheng H, Chattopadhyay R, et al. Multiple antigen peptide vaccines against Plasmodium falciparum malaria. Infect Immun. 2010;78(11):4613-24.

27. Van Regenmortel $\mathrm{MH}$. What is a B-cell epitope? Methods Mol Biol. 2009;524:3-20.

28. Gao J, Faraggi E, Zhou Y, Ruan J, Kurgan L. BEST: improved prediction of B-cell epitopes from antigen sequences. PLoS One. 2012;7(6):e40104

29. El-Kady IM. T-cell immunity in human chronic toxoplasmosis. J Egypt Soc Parasitol. 2011;41(1):17-28.

30. Langermans JA, Van der Hulst ME, Nibbering PH, Hiemstra PS, Fransen L, Van Furth R. IFN-gamma-induced L-arginine-dependent toxoplasmastatic activity in murine peritoneal macrophages is mediated by endogenous tumor necrosis factor-alpha. J Immunol. 1992;148(2):568-74.

31. Filisetti $D$, Candolfi E. Immune response to Toxoplasma gondii. Ann Ist Super Sanita. 2004:40(1):71-80.
32. Oldenhove $\mathrm{G}$, et al. Decrease of Foxp3+ Treg cell number and acquisition of effector cell phenotype during lethal infection. Immunity. 2009;31(5):772-86

33. Zheng B, Lu S, Tong Q, Kong Q, Lou D. The virulence-related rhoptry protein 5 (ROP5) of Toxoplasma gondii is a novel vaccine candidate against toxoplasmosis in mice. Vaccine. 2013:31(41):4578-84.

34. Gazzinelli RT, Wysocka M, Hieny S, Scharton-Kersten T, Cheever A, Kühn R, et al. In the absence of endogenous IL-10, mice acutely infected with Toxoplasma gondii succumb to a lethal immune response dependent on CD4+ T cells and accompanied by overproduction of IL-12. IFN-gamma and TNF-alpha J Immunol. 1996;157(2):798-805.

35. Dawson HD, Beshah E, Nishi S, Solano-Aguilar G, Morimoto M, Zhao A, et al. Localized multigene expression patterns support an evolving Th1/Th2-like paradigm in response to infections with Toxoplasma gondii and Ascaris suum. Infect Immun. 2005:73(2):1116-28.

36. Kang H, Remington JS, Suzuki Y. Decreased resistance of B cell-deficient mice to infection with Toxoplasma gondii despite unimpaired expression of IFN-gamma, TNF-alpha, and inducible nitric oxide synthase. J Immunol. 2000;164(5):2629-34.

37. Sayles PC, Gibson GW, Johnson LL. B cells are essential for vaccinationinduced resistance to virulent Toxoplasma gondii. Infect Immun. 2000;68(3):1026-33.

38. Zhang NZ, Xu Y, Wang M, Petersen E, Chen J, Huang SY, et al. Protective efficacy of two novel DNA vaccines expressing Toxoplasma gondii rhomboid 4 and rhomboid 5 proteins against acute and chronic toxoplasmosis in mice. Expert Rev Vaccines. 2015;14(9):1289-97.

39. Zhao G, Zhou A, Lu G, Meng M, Sun M, Bai Y, et al. Identification and characterization of Toxoplasma gondii aspartic protease 1 as a novel vaccine candidate against toxoplasmosis. Parasit Vectors. 2013;6:175.

40. Lambracht-Washington D, Qu BX, Fu M, Anderson Jr LD, Eagar TN, Stuve O, et al. A peptide prime-DNA boost immunization protocol provides significant benefits as a new generation Abeta42 DNA vaccine for Alzheimer disease. J Neuroimmunol. 2013:254(1-2):63-8.

41. Ciesielski MJ, Kazim AL, Barth RF, Fenstermaker RA. Cellular antitumor immune response to a branched lysine multiple antigenic peptide containing epitopes of a common tumor-specific antigen in a rat glioma model. Cancer Immunol Immunother. 2005:54(2):107-19.

42. Woodland DL. Jump-starting the immune system: prime-boosting comes of age. Trends Immunol.2004; 25(2):98-104

\section{Submit your next manuscript to BioMed Central} and we will help you at every step:

- We accept pre-submission inquiries

- Our selector tool helps you to find the most relevant journal

- We provide round the clock customer support

- Convenient online submission

- Thorough peer review

- Inclusion in PubMed and all major indexing services

- Maximum visibility for your research

Submit your manuscript at www biomedcentral com/submit
Biomed Central 